\title{
Chinese Syrian Gold Hamster
}

National Cancer Institute

\section{Source}

National Cancer Institute. Chinese Syrian Gold Hamster. NCI Thesaurus. Code C77093.

Derived from a cross between a Chinese hamster and a Syrian hamster, with a goldencolored coat. 